\title{
Oscillatory behavior of solutions of odd-order nonlinear delay differential equations
}

\section{Osama Moaaz ${ }^{1 *}$ (D)}

\section{"Correspondence:}

o_moaaz@mans.edu.eg

'Department of Mathematics,

Faculty of Science, Mansoura

University, 35516, Mansoura, Egypt

\begin{abstract}
The objective of this study is to establish new sufficient criteria for oscillation of solutions of odd-order nonlinear delay differential equations. Based on creating comparison theorems that compare the odd-order equation with a couple of first-order equations, we improve and complement a number of related ones in the literature. To show the importance of our results, we provide an example.
\end{abstract}

Keywords: Odd-order; Delay differential equations; Oscillatory behavior

\section{Introduction}

In this study, we investigate the oscillatory behavior of solutions of the odd-order delay differential equation (DDE)

$$
\left(r(t)\left(x^{(n-1)}(t)\right)^{\alpha}\right)^{\prime}+q(t) x^{\alpha}(\sigma(t))=0
$$

where $t \geq t_{0}, n \in \mathbb{Z}^{+}$is odd, $\alpha$ is a ratio of odd positive integers, $r \in C^{1}\left(\left[t_{0}, \infty\right),(0, \infty)\right)$, $r^{\prime}(t) \geq 0, \mu_{0,0}\left(t, t_{0}\right):=\int_{t_{0}}^{t} r^{-1 / \alpha}(s) \mathrm{d} s \rightarrow \infty$ as $t \rightarrow \infty, q \in C\left(\left[t_{0}, \infty\right),[0, \infty)\right), \sigma \in C\left(\left[t_{0}, \infty\right)\right.$, $\mathbb{R}), \sigma(t)<t$, and $\lim _{t \rightarrow \infty} \sigma(t)=\infty$.

Definition 1 Let $x \in C^{(n-1)}\left(\left[t_{x}, \infty\right)\right), t_{x} \geq t_{0}$, and $r\left(x^{(n-1)}\right)^{\alpha} \in C^{1}\left(\left[t_{x}, \infty\right)\right)$. The function $x$ is called a solution of (1.1) on $\left[t_{x}, \infty\right)$ if $x$ satisfies (1.1) for all $t$ in $\left[t_{x}, \infty\right)$.

Definition 2 A nontrivial solution $x$ of (1.1) is said to be oscillatory if there exists a sequence of zeros $\left\{t_{n}\right\}_{n=0}^{\infty}$ (i.e., $x\left(t_{n}\right)=0$ ) of $x$ such that $\lim _{n \rightarrow \infty} t_{n}=\infty$; otherwise, it is said to be nonoscillatory.

Although differential equations of even-order have been studied extensively, the study of qualitative behavior of odd-order differential equations has received considerably less attention in the literature, especially the third-order DDEs. However, certain results for third-order equations have been known for a long time and have some applications in

(c) The Author(s) 2020. This article is licensed under a Creative Commons Attribution 4.0 International License, which permits use, sharing, adaptation, distribution and reproduction in any medium or format, as long as you give appropriate credit to the original author(s) and the source, provide a link to the Creative Commons licence, and indicate if changes were made. The images or other third party material in this article are included in the article's Creative Commons licence, unless indicated otherwise in a credit line to the material. If material is not included in the article's Creative Commons licence and your intended use is not permitted by statutory regulation or exceeds the permitted use, you will need to obtain permission directly from the copyright holder. To view a copy of this licence, visit http://creativecommons.org/licenses/by/4.0/. 
mathematical modeling in biology and physics, see [17, 23, 25]. As a matter of fact, equation (1.1) under study is a so-called odd-order half-linear DDE, which has numerous applications in the research area of porous medium, see [13].

Different techniques have been used in studying the asymptotic behavior of DDEs. The articles $[1,3-9,14-16,27]$ were concerned with (in the canonical case and noncanonical case) the oscillation and asymptotic behavior of equation (1.1) and its special cases.

Based on creating comparison theorems that compare the odd-order DDEs with one or a couple of first-order DDEs, Agarwal et al. [1], Baculikova and Dzurina [3, 4] and Chatzarakis et al. [8] studied the oscillatory and asymptotic behavior of special cases of the third-order DDE

$$
\left(a(t)\left(\left(b(t) x^{\prime}(t)\right)^{\prime}\right)^{\alpha}\right)^{\prime}+q(t) f(x(\sigma(t)))=0
$$

where $a, b \in C^{1}\left(\left[t_{0}, \infty\right),(0, \infty)\right)$. By using the integral averaging technique, Bohner et al. [6] and Moaaz et al. [20] studied the asymptotic behavior of DDE with damping

$$
\left(a(t)\left(b(t)\left(x^{\prime}(t)\right)^{\alpha}\right)^{\prime}\right)^{\prime}+p(t)\left(x^{\prime}(t)\right)^{\alpha}+q(t) f(x(\sigma(t)))=0,
$$

where $\alpha \geq 1$ and $p \in C\left(\left[t_{0}, \infty\right),[0, \infty)\right)$. On the other hand, [5] used the Riccati transformation to study the asymptotic properties of the odd-order advanced equation

$$
\left(r(t)\left(x^{(n-1)}(t)\right)^{\alpha}\right)^{\prime}+q(t) x^{\alpha}(g(t))=0,
$$

where $g(t)>t$. The results concerned with the asymptotic properties and oscillation of the higher-order neutral DDEs were presented in [11, 18, 19, 21, 22, 26].

In this paper, by using an iterative method, we create sharper estimates for increasing and decreasing positive solutions of (1.1). Thus, we create sharper criteria for oscillation of (1.1). Moreover, iterative technique allows us to test the oscillation, even when the related results fail to apply. The results reported in this paper generalize, complement, and improve those in $[7-9,14-16,27]$. To show the importance of our results, we provide an example.

Remark 1.1 We restrict our discussion to those solutions $x$ of (1.1) which satisfy $\sup \{|x(t)|$ : $t \geq T\}>0$ for every $T \in\left[t_{0}, \infty\right)$.

Remark 1.2 All functional inequalities and properties, such as increasing, decreasing, positive, and so on, are assumed to hold eventually, that is, they are satisfied for all $t$ large enough.

\section{Main results}

Lemma 2.1 ([2, Lemma 2.2.3]) Let $F \in C^{n}\left(\left[t_{0}, \infty\right),(0, \infty)\right), F^{(n-1)}(t) F^{(n)}(t) \leq 0$ for $t \geq t_{F}$, and $\lim _{t \rightarrow \infty} F(t) \neq 0$. Then, for every $\delta \in(0,1)$, there exists $t_{\delta} \in\left[t_{F}, \infty\right)$ such that

$$
F(t) \geq \frac{\delta}{(n-1) !} t^{n-1}\left|F^{(n-1)}(t)\right| \quad \text { for all } t \in\left[t_{\delta}, \infty\right)
$$


Lemma 2.2 ([5, Lemma 2]) If $x$ is a positive solution of $(1.1)$, then all derivatives $x^{(k)}(t), 1 \leq$ $k \leq n-1$, are of constant signs, $r(t)\left(x^{(n-1)}(t)\right)^{\alpha}$ is nonincreasing, and $x$ satisfies either

$$
x^{\prime}(t)>0, \quad x^{\prime \prime}(t)>0, \quad x^{(n-1)}(t)>0, \quad x^{(n)}(t)<0
$$

or

$$
(-1)^{m} x^{(m)}>0, \quad m=1,2, \ldots, n .
$$

Definition 3 The set of all positive solutions of (1.1) with property (2.1) or (2.2) is denoted by $X_{I}^{+}$or $X_{D}^{+}$, respectively.

Lemma 2.3 Assume that $x \in X_{I}^{+}$. Then

$$
x(\sigma(t)) \geq \eta_{k}(\sigma(t)) x^{(n-1)}(\sigma(t)),
$$

where

$$
\eta_{0}(t):=\frac{\delta_{0}}{(n-1) !} t^{n-1}
$$

and

$$
\eta_{k+1}(t):=\frac{\delta_{k}}{(n-2) !} r^{1 / \alpha}(t) \int_{t_{1}}^{t} s^{n-2}\left(\frac{1}{r(s)} \exp \left(\int_{s}^{t} \frac{1}{r(u)} q(u) \eta_{k}^{\alpha}(\sigma(u)) \mathrm{d} u\right)\right)^{1 / \alpha} \mathrm{d} s
$$

for all $\delta_{k} \in(0,1)$ and $k=0,1, \ldots$

Proof Let $x \in X_{I}^{+}$. Then there exists $t_{1} \geq t_{0}$ such that $x(t)>0$ and $x(\sigma(t))>0$ for all $t \geq t_{1}$. Next, we will prove (2.3) using induction. For $k=0$, using Lemma 2.1, we see that

$$
x(\sigma(t)) \geq \frac{\delta_{0}}{(n-1) !} \sigma^{n-1}(t) x^{(n-1)}(\sigma(t)) \geq \eta_{0}(\sigma(t)) x^{(n-1)}(\sigma(t)) .
$$

Now, we assume that $x(\sigma(t)) \geq \eta_{k}(\sigma(t)) x^{(n-1)}(\sigma(t))$ for $k>0$. Since $x^{(n)}<0$ and $\sigma(t)<t$, we have that

$$
x(\sigma(t)) \geq \eta_{k}(\sigma(t)) x^{(n-1)}(t) .
$$

Then, from (1.1) and (2.4), we get

$$
\left(r(t)\left(x^{(n-1)}(t)\right)^{\alpha}\right)^{\prime}+q(t) \eta_{k}^{\alpha}(\sigma(t))\left(x^{(n-1)}(t)\right)^{\alpha} \leq 0
$$

If we set $w:=r(t)\left(x^{(n-1)}(t)\right)^{\alpha}$, then (2.5) becomes

$$
w^{\prime}(t) \leq-\frac{1}{r(t)} q(t) \eta_{k}^{\alpha}(\sigma(t)) w(t)
$$


Applying the Grönwall inequality, we find

$$
w(s) \geq w(t) \exp \left(\int_{s}^{t} \frac{1}{r(u)} q(u) \eta_{k}^{\alpha}(\sigma(u)) \mathrm{d} u\right)
$$

or

$$
x^{(n-1)}(s) \geq r^{1 / \alpha}(t) x^{(n-1)}(t)\left(\frac{1}{r(s)} \exp \left(\int_{s}^{t} \frac{1}{r(u)} q(u) \eta_{k}^{\alpha}(\sigma(u)) \mathrm{d} u\right)\right)^{1 / \alpha} .
$$

Using Lemma 2.1 with $F:=x^{\prime}>0$, we see that

$$
x^{\prime}(t) \geq \frac{\delta_{k} t^{n-2}}{(n-2) !} x^{(n-1)}(t) \quad \text { for all } \delta_{k} \in(0,1)
$$

By integrating this inequality from $t_{1}$ to $t$ and taking into account (2.6), we see that

$$
\begin{aligned}
x(t) & \geq \frac{\delta_{k}}{(n-2) !} \int_{t_{1}}^{t} s^{n-2} x^{(n-1)}(s) \mathrm{d} s \\
& \geq x^{(n-1)}(t) \frac{\delta_{k}}{(n-2) !} r^{1 / \alpha}(t) \int_{t_{1}}^{t} s^{n-2}\left(\frac{1}{r(s)} \exp \left(\int_{s}^{t} \frac{1}{r(u)} q(u) \eta_{k}^{\alpha}(\sigma(u)) \mathrm{d} u\right)\right)^{1 / \alpha} \mathrm{d} s \\
& \geq \eta_{k+1}(t) x^{(n-1)}(t) .
\end{aligned}
$$

Therefore, we have that

$$
x(\sigma(t)) \geq \eta_{k+1}(\sigma(t)) x^{(n-1)}(\sigma(t)) .
$$

The proof is complete.

Lemma 2.4 Assume that $x \in X_{D}^{+}$. Then

$$
x(u) \geq r^{1 / \alpha}(v) x^{(n-1)}(v) \mu_{l, n-2}(v, u),
$$

where

$$
\mu_{l, k+1}(v, u):=\int_{u}^{v} \mu_{l, k}(v, s) \mathrm{d} s
$$

and

$$
\mu_{l+1,0}(v, u):=\int_{u}^{v} \frac{1}{r^{1 / \alpha}(s)} \exp \left(\int_{s}^{v} q(u) \mu_{l, n-2}^{\alpha}(u, \sigma(u)) \mathrm{d} u\right)^{1 / \alpha} \mathrm{d} s
$$

for $k=0,1, \ldots, n-3$, and $l=0,1,2, \ldots$

Proof Let $x \in X_{D}^{+}$. Then there exists $t_{1} \geq t_{0}$ such that $x(t)>0$ and $x(\sigma(t))>0$ for all $t \geq t_{1}$. Next, we will prove (2.7) using induction. For $l=0$, since $\left(r\left(z^{(n-1)}\right)\right)^{\prime} \leq 0$, we get that

$$
-x^{(n-2)}(u) \geq x^{(n-2)}(v)-x^{(n-2)}(u)=\int_{u}^{v} \frac{1}{r^{1 / \alpha}(s)} r^{1 / \alpha}(s) x^{(n-1)}(s) \mathrm{d} s
$$




$$
\geq r^{1 / \alpha}(v) x^{(n-1)}(v) \mu_{0,0}(v, u)
$$

Integrating (2.8) from $u$ to $v$, we have

$$
-x^{(n-3)}(u) \leq x^{(n-3)}(v)-x^{(n-3)}(u)=r^{1 / \alpha}(v) x^{(n-1)}(v) \mu_{0,1}(v, u) .
$$

Integrating (2.9) $n-3$ times from $u$ to $v$, we get

$$
x(u) \geq r^{1 / \alpha}(v) x^{(n-1)}(v) \mu_{0, n-2}(v, u) .
$$

Now, we assume that $x(u) \geq r^{1 / \alpha}(v) x^{(n-1)}(v) \mu_{l, n-2}(v, u)$ for $l>0$. Thus, we find

$$
x(\sigma(t)) \geq r^{1 / \alpha}(t) x^{(n-1)}(t) \mu_{l, n-2}(t, \sigma(t))
$$

which, with (1.1), gives

$$
\left(r(t)\left(x^{(n-1)}(t)\right)^{\alpha}\right)^{\prime}+q(t) r(t)\left(x^{(n-1)}(t)\right)^{\alpha} \mu_{l, n-2}^{\alpha}(t, \sigma(t)) \leq 0 .
$$

If we set $\psi:=r(t)\left(x^{(n-1)}(t)\right)^{\alpha}$, then (2.10) becomes

$$
\psi^{\prime}(t) \leq-q(t) \mu_{l, n-2}^{\alpha}(t, \sigma(t)) \psi(t) .
$$

Applying the Grönwall inequality, we find

$$
\psi(s) \geq \psi(v) \exp \left(\int_{s}^{v} q(u) \mu_{l, n-2}^{\alpha}(u, \sigma(u)) \mathrm{d} u\right)
$$

or

$$
x^{(n-1)}(s) \geq r^{1 / \alpha}(v) x^{(n-1)}(v)\left(\frac{1}{r(s)} \exp \left(\int_{s}^{v} q(u) \mu_{l, n-2}^{\alpha}(u, \sigma(u)) \mathrm{d} u\right)\right)^{1 / \alpha}
$$

Thus, from (2.8), we see that

$$
\begin{aligned}
-x^{(n-2)}(u) & \geq r^{1 / \alpha}(v) x^{(n-1)}(v) \int_{u}^{v} \frac{1}{r^{1 / \alpha}(s)} \exp \left(\int_{s}^{v} q(u) \mu_{l, n-2}^{\alpha}(u, \sigma(u)) \mathrm{d} u\right)^{1 / \alpha} \mathrm{d} s \\
& \geq r^{1 / \alpha}(v) x^{(n-1)}(v) \mu_{l+1,0}(v, u) .
\end{aligned}
$$

Integrating this inequality $n-2$ times from $u$ to $v$, we get

$$
x(u) \geq r^{1 / \alpha}(v) x^{(n-1)}(v) \mu_{l+1, n-2}(v, u) .
$$

Thus, the proof is complete.

Theorem 2.1 Assume that $x$ is a positive solution of (1.1) and $\eta_{k}$ is defined as in Lemma 2.3. If the delay differential equation

$$
w^{\prime}(t)+\frac{1}{r(\sigma(t))} q(t) \eta_{k}^{\alpha}(\sigma(t)) w(\sigma(t))=0
$$

is oscillatory for some $\delta_{k} \in(0,1)$ and some $k \in \mathbb{N}$, then $X_{I}^{+}$is empty. 
Proof Assume to the contrary that $x \in X_{I}^{+}$. Then there exists $t_{1} \geq t_{0}$ such that $x(t)>0$ and $x(\sigma(t))>0$ for all $t \geq t_{1}$. From Lemma 2.3, we have that (2.3) holds. Combining (1.1) and (2.3), we obtain

$$
\left(r(t)\left(x^{(n-1)}(t)\right)^{\alpha}\right)^{\prime}+q(t) \eta_{k}^{\alpha}(\sigma(t))\left(x^{(n-1)}(\sigma(t))\right)^{\alpha} \leq 0 .
$$

If we set $w:=r\left(x^{(n-1)}\right)^{\alpha}$, then (2.12) becomes

$$
w^{\prime}(t)+\frac{1}{r(\sigma(t))} q(t) \eta_{k}^{\alpha}(\sigma(t)) w(\sigma(t)) \leq 0 .
$$

In view of [24, Theorem 1], we have that (2.11) also has a positive solution, a contradiction. Thus, the proof is complete.

Corollary 2.1 Assume that $x$ is a positive solution of (1.1) and $\eta_{k}$ is defined as in Lemma 2.3. If

$$
\liminf _{t \rightarrow \infty} \int_{\sigma(t)}^{t} \frac{1}{r(\sigma(u))} q(u) \eta_{k}^{\alpha}(\sigma(u)) \mathrm{d} u>\frac{1}{\mathrm{e}}
$$

for some $\delta_{k} \in(0,1)$ and some $k \in \mathbb{N}$, then $X_{I}^{+}$is empty.

Proof In view of [12, Theorem 2], condition (2.13) guarantees that the delay equation (2.11) is oscillatory.

Theorem 2.2 Assume that $x$ is a positive solution of $(1.1), \sigma^{\prime}(t)>0$, and $\mu_{l, k}$ is defined as in Lemma 2.4. If

$$
\limsup _{t \rightarrow \infty} \int_{\sigma(t)}^{t} q(u) \mu_{l, n-2}^{\alpha}(\sigma(t), \sigma(u)) \mathrm{d} u>1
$$

for some $l \in \mathbb{N}$, then $X_{D}^{+}$is empty.

Proof Assume to the contrary that $x \in X_{D}^{+}$. Then there exists $t_{1} \geq t_{0}$ such that $x(t)>0$ and $x(\sigma(t))>0$ for all $t \geq t_{1}$. From Lemma 2.4, we have that (2.7) holds. Integrating (1.1) from $\sigma(t)$ to $t$, we obtain

$$
r(\sigma(t))\left(x^{(n-1)}(\sigma(t))\right)^{\alpha}-r(t)\left(x^{(n-1)}(t)\right)^{\alpha}=\int_{\sigma(t)}^{t} q(u) x^{\alpha}(\sigma(u)) \mathrm{d} u,
$$

and so

$$
r(\sigma(t))\left(x^{(n-1)}(\sigma(t))\right)^{\alpha} \geq \int_{\sigma(t)}^{t} q(u) x^{\alpha}(\sigma(u)) \mathrm{d} u .
$$

Using (2.7) with $u=\sigma(u)$ and $v=\sigma(t)$, we get that

$$
x(\sigma(u)) \geq r^{1 / \alpha}(\sigma(t)) x^{(n-1)}(\sigma(t)) \mu_{l, n-2}(\sigma(t), \sigma(u)),
$$


with (2.15), gives

$$
\int_{\sigma(t)}^{t} q(u) \mu_{l, n-2}^{\alpha}(\sigma(t), \sigma(u)) \mathrm{d} u \leq 1
$$

which contradicts condition (2.14). This completes the proof.

Theorem 2.3 Assume that $x$ is a positive solution of (1.1) and $\mu_{l, k}$ is defined as in Lemma 2.4. If there exists a function $\theta \in C\left(\left[t_{0}, \infty\right),(0, \infty)\right)$ satisfying $\theta(t)<t$ and $\sigma(t)<\theta(t)$ such that the delay differential equation

$$
\varphi^{\prime}(t)+q(t) \mu_{l, n-2}^{\alpha}(\theta(t), \sigma(t)) \varphi(\theta(t))=0
$$

is oscillatory for some $l \in \mathbb{N}$, then $X_{D}^{+}$is empty.

Proof Assume to the contrary that $x \in X_{D}^{+}$. Then there exists $t_{1} \geq t_{0}$ such that $x(t)>0$ and $x(\sigma(t))>0$ for all $t \geq t_{1}$. From Lemma 2.4, we have that (2.7) holds. Using (2.7) with $u=\sigma(t)$ and $v=\theta(t)$, we get that

$$
x(\sigma(t)) \geq r^{1 / \alpha}(\theta(t)) x^{(n-1)}(\theta(t)) \mu_{l, n-2}(\theta(t), \sigma(t)) .
$$

Thus, from (1.1), we obtain

$$
\left(r(t)\left(x^{(n-1)}(t)\right)^{\alpha}\right)^{\prime}+q(t) \mu_{l, n-2}^{\alpha}(\theta(t), \sigma(t)) r(\theta(t))\left(x^{(n-1)}(\theta(t))\right)^{\alpha} \leq 0 .
$$

If we set $\varphi:=r\left(x^{(n-1)}\right)^{\alpha}$, then $(2.17)$ becomes

$$
\varphi^{\prime}(t)+q(t) \mu_{l, n-2}^{\alpha}(\theta(t), \sigma(t)) \varphi(\theta(t)) \leq 0 .
$$

In view of [24, Theorem 1], we have that (2.16) also has a positive solution, a contradiction. Thus, the proof is complete.

Theorem 2.4 Assume that $x$ is a positive solution of $(1.1),\left(\sigma^{-1}(t)\right)^{\prime}>0$ and $\mu_{l, k}$ is defined as in Lemma 2.4. If there exists a function $\vartheta \in C\left(\left[t_{0}, \infty\right),(0, \infty)\right)$ satisfying $\vartheta(t)>t$ and $\sigma(\vartheta(t))<t$ such that the delay differential equation

$$
\varphi^{\prime}(t)+\left(\sigma^{-1}(t)\right)^{\prime} q\left(\sigma^{-1}(t)\right) \mu_{l, n-2}^{\alpha}(\vartheta(t), t) \varphi(\sigma(\vartheta(t)))=0
$$

is oscillatory for some $l \in \mathbb{N}$, then $X_{D}^{+}$is empty.

Proof Assume to the contrary that $x \in X_{D}^{+}$. Then there exists $t_{1} \geq t_{0}$ such that $x(t)>0$ and $x(\sigma(t))>0$ for all $t \geq t_{1}$. From Lemma 2.4, we have that (2.7) holds. From (1.1), we get

$$
\left(r\left(\sigma^{-1}(t)\right)\left(x^{(n-1)}\left(\sigma^{-1}(t)\right)\right)^{\alpha}\right)^{\prime}+\left(\sigma^{-1}(t)\right)^{\prime} q\left(\sigma^{-1}(t)\right) x^{\alpha}(t)=0 .
$$

Using (2.7) with $u=t$ and $v=\vartheta(t)$, we have

$$
x(t) \geq r^{1 / \alpha}(\vartheta(t)) x^{(n-1)}(\vartheta(t)) \mu_{l, n-2}(\vartheta(t), t),
$$


which with (2.19) gives

$$
\begin{aligned}
0 \geq & \left(r\left(\sigma^{-1}(t)\right)\left(x^{(n-1)}\left(\sigma^{-1}(t)\right)\right)^{\alpha}\right)^{\prime} \\
& +\left(\sigma^{-1}(t)\right)^{\prime} q\left(\sigma^{-1}(t)\right) \mu_{l, n-2}^{\alpha}(\vartheta(t), t) r(\vartheta(t))\left(x^{(n-1)}(\vartheta(t))\right)^{\alpha} .
\end{aligned}
$$

If we set $\varphi(t):=r\left(x^{(n-1)}\right)^{\alpha}\left(\sigma^{-1}(t)\right)$, then (2.20) becomes

$$
\varphi^{\prime}(t)+\left(\sigma^{-1}(t)\right)^{\prime} q\left(\sigma^{-1}(t)\right) \mu_{l, n-2}^{\alpha}(\vartheta(t), t) \varphi(\sigma(\vartheta(t))) \leq 0 .
$$

In view of [24, Theorem 1], we have that (2.18) also has a positive solution, a contradiction. Thus, the proof is complete.

Applying a well-known criterion [12, Theorem 2] for delay equations (2.16) and (2.18) to be oscillatory, we obtain the following two corollaries.

Corollary 2.2 Assume that $x$ is a positive solution of (1.1) and $\mu_{l, k}$ is defined as in Lemma 2.4. If there exists a function $\theta \in C\left(\left[t_{0}, \infty\right),(0, \infty)\right)$ satisfying $\theta(t)<t$ and $\sigma(t)<\theta(t)$ such that

$$
\liminf _{t \rightarrow \infty} \int_{\theta(t)}^{t} q(u) \mu_{l, n-2}^{\alpha}(\theta(u), \sigma(u)) \mathrm{d} u>\frac{1}{\mathrm{e}}
$$

for some $l \in \mathbb{N}$, then $X_{D}^{+}$is empty.

Corollary 2.3 Assume that $x$ is a positive solution of $(1.1),\left(\sigma^{-1}(t)\right)^{\prime}>0$ and $\mu_{l, k}$ is defined as in Lemma 2.4. If there exists a function $\vartheta \in C\left(\left[t_{0}, \infty\right),(0, \infty)\right)$ satisfying $\vartheta(t)>t$ and $\sigma(\vartheta(t))<t$ such that

$$
\liminf _{t \rightarrow \infty} \int_{\sigma(\vartheta(t))}^{t}\left(\sigma^{-1}(u)\right)^{\prime} q\left(\sigma^{-1}(u)\right) \mu_{l, n-2}^{\alpha}(\vartheta(u), u) \mathrm{d} u>\frac{1}{\mathrm{e}}
$$

for some $l \in \mathbb{N}$, then $X_{D}^{+}$is empty.

Theorem 2.5 Assume that $\eta_{k}$ and $\mu_{l, k}$ are defined as in Lemmas 2.3 and 2.4, respectively. Then every solution of (1.1) is oscillatory if one of the following conditions is satisfied for some $\delta_{k} \in(0,1)$ and some $k, l \in \mathbb{N}$ :

(a) There exists a function $\theta \in C\left(\left[t_{0}, \infty\right),(0, \infty)\right)$ satisfying $\theta(t)<t$ and $\sigma(t)<\theta(t)$ such that the delay differential equations (2.11) and (2.16) are oscillatory;

(b) There exists a function $\vartheta \in C\left(\left[t_{0}, \infty\right),(0, \infty)\right)$ satisfying $\vartheta(t)>t,\left(\sigma^{-1}(t)\right)^{\prime}>0$ and $\sigma(\vartheta(t))<t$ such that the delay differential equations (2.11) and (2.18) are oscillatory.

Corollary 2.4 Assume that $\eta_{k}$ and $\mu_{l, k}$ are defined as in Lemmas 2.3 and 2.4, respectively. Then every solution of (1.1) is oscillatory if one of the following conditions is satisfied for some $\delta_{k} \in(0,1)$ and some $k, l \in \mathbb{N}$ :

(a) Conditions (2.13) and (2.14) hold;

(b) Conditions (2.13) and (2.21) hold;

(c) Conditions (2.13) and (2.22) hold. 
Remark 2.1 The article [10] was concerned with the oscillation of equations (2.11), (2.16), and (2.18). Thus, one can obtain a number of oscillation criteria for (1.1) by using related results reported in [10].

Example 2.1 Consider the third-order differential equation

$$
x^{\prime \prime \prime}+\frac{q_{0}}{t^{3}} x(\lambda t)=0,
$$

where $t \geq 1, q_{0}>0$, and $\lambda \in(0,2 / 3)$. It is easy to verify that $\eta_{0}(t):=\frac{\delta_{0}}{2} \lambda^{2} t^{2}, \mu_{0,0}(v, u)=v-u$, $\mu_{0,1}(v, u)=\frac{1}{2}(v-u)^{2}$,

$$
\mu_{1,0}(v, u)=q_{0} \frac{(1-\lambda)^{2}}{2} v \ln \frac{v}{u}
$$

and

$$
\mu_{1,1}(v, u)=q_{0} \frac{(1-\lambda)^{2}}{2} v\left(v-u\left(1+\ln \frac{v}{u}\right)\right) .
$$

Thus, by choosing $k=0, l=1$ and $\theta(t):=\frac{3}{2} \lambda t$, conditions (2.13) and (2.21) reduce to

$$
q_{0} \lambda^{2} \ln \frac{1}{\lambda}>\frac{2}{\mathrm{e}}
$$

and

$$
q_{0}^{2} \frac{3}{4} \lambda^{2}(\lambda-1)^{2}\left(\frac{1}{2}-\ln \frac{3}{2}\right) \ln \frac{2}{3 \lambda}>\frac{1}{\mathrm{e}}
$$

respectively. Using Corollary 2.4(b), we see that every solution of (2.23) is oscillatory if (2.24) and (2.25) hold.

Remark 2.2 Apparently, Corollary 2.4(a) and Theorem 2 in [8] are the same for $n=3$. Consider a particular case of (2.23), namely $x^{\prime \prime \prime}+q_{0} t^{-3} x(0.5 t)=0$. By using the results in Example 2.1, this equation is oscillatory if $q_{0}>16.988$.

\section{Acknowledgements}

The author presents their sincere thanks to the editors.

Funding

For this paper, no direct funding was received.

Availability of data and materials

No data sharing (where no datasets are produced).

Competing interests

There are no competing interests.

Author's contributions

All authors read and approved the final manuscript.

\section{Publisher's Note}

Springer Nature remains neutral with regard to jurisdictional claims in published maps and institutional affiliations.

Received: 26 May 2020 Accepted: 6 July 2020 Published online: 14 July 2020 


\section{References}

1. Agarwal, R.P., Bohner, M., Li, T., Zhang, C.: Oscillation of third-order nonlinear delay differential equations. Taiwan. J. Math. 17(2), 545-558 (2013)

2. Agarwal, R.P., Grace, S.R., O'Regan, D.: Oscillation Theory for Difference and Functional Differential Equations. Dekker, Dordrecht (2000)

3. Baculikova, B., Dzurina, J.: Oscillation of third-order functional differential equations. Electron. J. Qual. Theory Differ. Equ. 43, 1 (2010)

4. Baculikova, B., Dzurina, J.: Oscillation of third-order nonlinear differential equations. Appl. Math. Lett. 24(4), 466-470 (2011)

5. Baculikova, B., Dzurina, J.: On the oscillation of odd order advanced differential equations. Bound. Value Probl. 2014, 214 (2014)

6. Bohner, M., Grace, S.R., Sager, I., Tunc, E.: Oscillation of third-order nonlinear damped delay differential equations. Appl. Math. Comput. 278, 21-32 (2016)

7. Chatzarakis, G.E., Dzurina, J., Jadlovska, I.: Oscillatory and asymptotic properties of third-order quasilinear delay differential equations. J. Inequal. Appl. 2019, Article ID 23 (2019)

8. Chatzarakis, G.E., Grace, S.R., Jadlovska, l.: Oscillation criteria for third-order delay differential equations. Adv. Differ. Equ. 2017, 330 (2017)

9. Chatzarakis, G.E., Grace, S.R., Jadlovska, I., Li, T., Tunc, T.: Oscillation criteria for third-order Emden-Fowler differential equations with unbounded neutral coefficients. Complexity 2019, Article ID 5691758 (2019)

10. Chatzarakis, G.E., Li, T.: Oscillation criteria for delay and advanced differential equations with nonmonotone arguments. Complexity 2018, Article ID 8237634 (2018)

11. Dzurina, J., Grace, S.R., Jadlovska, I.: On nonexistence of Kneser solutions of third-order neutral delay differential equations. Appl. Math. Lett. 88, 193-200 (2019)

12. Kitamura, Y., Kusano, T.: Oscillation of first-order nonlinear differential equations with deviating arguments. Proc. Am. Math. Soc. 78(1), 64-68 (1980)

13. Li, T., Pintus, N., Viglialoro, G.: Properties of solutions to porous medium problems with different sources and boundary conditions. Z. Angew. Math. Phys. 70, Article ID 86 (2019)

14. Li, T., Rogovchenko, Y.V.: Asymptotic behavior of higher-order quasilinear neutral differential equations. Abstr. Appl. Anal. 2014, Article ID 395368 (2014)

15. Li, T., Rogovchenko, Y.V.: On asymptotic behavior of solutions to higher-order sublinear Emden-Fowler delay differential equations. Appl. Math. Lett. 67, 53-59 (2017)

16. Li, T., Rogovchenko, Y.V.: On the asymptotic behavior of solutions to a class of third-order nonlinear neutral differentia equations. Appl. Math. Lett. 105, Article ID 106293 (2020)

17. McKean, H.P.: Nagumo's equation. Adv. Math. 4(3), 209-223 (1970)

18. Moaaz, O., Baleanu, D., Muhib, A.: New aspects for non-existence of Kneser solutions of neutral differential equations with odd-order. Mathematics 8(4), 494 (2020)

19. Moaaz, O., Chalishajar, D., Bazighifan, O.: Asymptotic behavior of solutions of the third order nonlinear mixed type neutral differential equations. Mathematics 8(4), 485 (2020)

20. Moaaz, O., Elabbasy, E.M., Shaaban, E.: Oscillation criteria for a class of third order damped differential equations. Arab J. Math. Sci. 24(1), 16-30 (2018)

21. Moaaz, O., Muhib, A.: New oscillation criteria for nonlinear delay differential equations of fourth-order. Appl. Math Comput. 377, $125192(2020)$

22. Moaaz, O., Qaraad, B., El-Nabulsi, R.A., Bazighifan, O.: New results for Kneser solutions of third-order nonlinear neutral differential equations. Mathematics 8(5), 686 (2020)

23. Padhi, S., Pati, S.: Theory of Third-Order Differential Equations. Springer, New Delhi (2014)

24. Philos, C:: On the existence of nonoscillatory solutions tending to zero at $\infty$ for differential equations with positive delays. Arch. Math. (Basel) 36(2), 168-178 (1981)

25. Vreeke, S.A., Sandquist, G.M.: Phase space analysis of reactor kinetics. Nucl. Sci. Eng. 42(3), $295-305$ (1970)

26. Xing, G., Li, T., Zhang, C.: Oscillation of higher-order quasi-linear neutral differential equations. Adv. Differ. Equ. 2011, 45 (2011)

27. Zhang, C., Li, T., Sun, B., Thandapani, E.: On the oscillation of higher-order half-linear delay differential equations. Appl. Math. Lett. 24(9), 1618-1621 (2011)

\section{Submit your manuscript to a SpringerOpen ${ }^{\circ}$ journal and benefit from:}

- Convenient online submission

- Rigorous peer review

- Open access: articles freely available online

- High visibility within the field

- Retaining the copyright to your article

Submit your next manuscript at $>$ springeropen.com 